\title{
Las alteraciones silíceas de las lavas de Montaña Señalo, erupción de Timanfaya (1730-1736) (Lanzarote, Islas Canarias)
}

\author{
Siliceous alterations of the Montaña Señalo lavas, \\ Timanfaya eruption (1730-1736) (Lanzarote, Canary Islands)
}

\author{
J. Carmona ${ }^{1}$, C. Romero², J. Dóniz³, A. García ${ }^{1}$
}

RESUMEN

La presencia de alteraciones de origen hidrotermal entre las lavas de la erupción de Timanfaya (1730-36), con altas proporciones del cuarzo y ópalo, indica la circulación efectiva de fluidos calientes. El origen de estos fluidos estaría en zonas profundas por debajo de la isla, donde se produciría la disolución de la sílice a partir de areniscas y radiolaritas, movilizándose de esta manera este componente hacia la superficie en forma de coloides de $\mathrm{Si}(\mathrm{OH})_{4}$. El estudio en profundidad del ópalo indica la presencia de fases tipo A-inicio de CT y C. Si se tiene en cuenta el tiempo en el que se producen estas transformaciones en la evolución diagenética de esta fase amorfa (10.000-50.000 años), indicaría la presencia de un proceso acelerador que podría estar relacionado con una importante circulación de fluidos o con procesos de alteración meteórica capaces, de producir estas transformaciones en unas lavas de menos de 300 años.

Palabras clave: alteración hidrotermal, ópalo-A, ópalo-C, sistema hidrológico, erupción Timanfaya, Lanzarote.

\begin{abstract}
The presence of hydrothermal alterations within the lavas of Timanfaya eruption (1730-1736), with high proportions of quartz and opal, suggests the effective circulation of hot fluids. The source of these fluids would be located under the island, where silica would be dissolved from sandstones and radiolarites, moving this way towards the surface as $\mathrm{Si}(\mathrm{OH})_{4}$ colloids. Study of opal indicates the presence of Ainitial CT and C phases in the collected samples which, considering the time needed for producing this phase transformations in the diagenetic evolution of opal (10,000-50,000 years), suggests an accelerating process, probably related with either the presence of fluid circulation or weathering processes. Such circumstances are necessary for explaining the presence of such components affecting 300 years old lavas.
\end{abstract}

Key words: hydrothermal alteration, opal-A, opal-C, hydrological system, Timanfaya eruption, Lanzarote.

\section{Introducción}

Para la formación de un depósito hidrotermal, es necesario por un lado una fuente de calor y por otro una circulación eficiente de fluidos, capaces de movilizar componentes de las rocas por las que atraviesa hacia otros lugares. Si estas aguas son capaces de alcanzar la superficie y contienen la carga química suficiente, las nuevas condiciones termodinámicas hacen que se puedan generar depósitos y alteraciones características, identificables tanto texturalmente como en composición y contenido mineral. Los depósitos más comunes son travertinos de composición carbonatada y sinters silíceos, que suelen

\footnotetext{
1 Departamento de Volcanología, Museo Nacional de Ciencias Naturales-CSIC, José Gutiérrez Abascal, 2, 28006 Madrid (España). Email: jcarmona@mncn.csic.es, aliciag@mncn.csic.es

2 Dpto. de Geografía, Universidad de La Laguna, Campus de Guajara s/n, 38071 La Laguna, Tenerife. Email: mcromero@ull.es

${ }^{3}$ Escuela de Turismo Iriarte, Universidad de La Laguna, Paseo Sto. Tomás, s/n, 38400, Puerto de La Cruz. Tenerife. Email: jdoniz@ull.es
} 


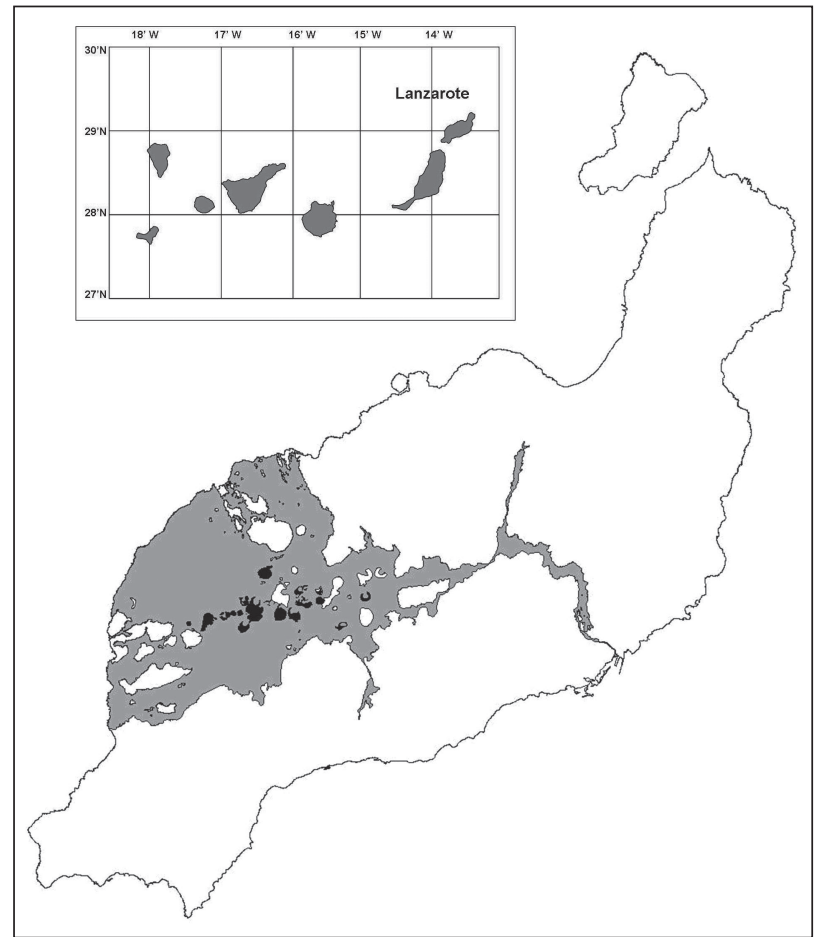

Fig. 1.-Localización de la isla de Lanzarote y la superficie afectada por las erupciones de 1730-36 y 1824 (gris) donde se emitieron gran cantidad de lavas desde sus centros eruptivos (negro) a lo largo de una fisura de más de $14 \mathrm{~km}$.

estar asociados a zonas de anomalías geotérmicas, activas en la actualidad o en el pasado, y que generalmente son originadas por fluidos de naturaleza próxima a la neutral de tipo clorurado alcalino, derivados de reservorios a más de $175{ }^{\circ} \mathrm{C}$ (Fournier \& Rowe, 1966). De esta manera, la presencia de zonas con gradientes geotérmicos anómalos y posibilidad de circulación de aguas subterráneas calientes deben tenerse muy en cuenta a la hora de buscar depósitos y/o alteraciones hidrotermales.

Las anomalías geotérmicas en las islas Canarias no son especialmente abundantes, localizándose puntualmente en la isla de Lanzarote, asociadas a una red de fracturación, y al cráter del Teide, en Tenerife, vinculadas con la desgasificación del propio estratovolcán. El área de estudio del presente trabajo ha sido la isla de Lanzarote, concretamente en la zona de Montaña Señalo, un volcán monogénico generado durante la erupción de Timanfaya (1730-1736).

\section{Marco geológico}

Lanzarote es la isla más al norte y al este del archipiélago canario (fig. 1), distando escasos $100 \mathrm{~km}$ del continente africano. Está compuesta por varios islotes localizados todos al norte y que representan, en conjunto, una extensión de $846 \mathrm{~km}^{2}$ aproximadamente.

En cuanto a su composición y estructura, es una isla volcánica de composición esencialmente basáltica, que pudo comenzar su actividad submarina ya en el Oligoceno, que es lo que hoy se reconoce en superficie como el basamento de la isla de Fuerteventura, formado por rocas plutónicas y sedimentos oceánicos, además de varios enjambres de diques inyectados en distintas direcciones. La emisión subaérea se divide en dos etapas separadas por una importante interrupción en la actividad eruptiva. Se reconoce una primera etapa donde se formarían los macizos de los Ajaches y de Famara localizados al SO y NE respectivamente, entre 15 y 3,8 Ma (Coello et al., 1992, Ibarrola et al., 1988, Abdel Monem et al., 1971) y una segunda correspondiente con la actividad reciente PlioCuaternaria, donde se generarían una serie de edificios estrombolianos de diversa envergadura, a favor de ejes volcanoestructurales en dirección NE-SO, que comenzaría su actividad hace 2 Ma y se prolongaría hasta las últimas erupciones acontecidas en 1730-1736 y 1824.

La erupción de Timanfaya, desarrollada en la isla de Lanzarote hacia mediados del siglo XVIII (1-091730 a 16-04-1736), dio lugar a la construcción de un sistema volcánico de gran complejidad morfológica y estructural. Durante la misma se abrieron más de 190 bocas cratéricas y se construyeron 27 conos volcánicos monogénicos, que se articulan en torno a una fractura principal de rumbo ENE-OSO de unos $13 \mathrm{~km}$ de longitud y a fracturas secundarias de rumbos NO-SE y N-S (Romero, 1991). La primera cartografía y estratigrafía corresponde con los trabajos de Fuster et al. (1968), completándose posteriormente por Carracedo et al. (1990) y Carracedo \& Badiola (1991) que realizan un cartografía en detalle sobre la extensión de esta erupción, además de una reconstrucción de los periodos eruptivos. Igualmente en este último trabajo se hace una división dentro de este evento volcánico en función del quimismo de las rocas, así se distingue una primera etapa donde se generan basanitas nefelínicas que evoluciona para producir, en una segunda fase, toleítas olivínicas. Este cambio en el quimismo lo relacionan con un proceso de somerización en la fuente magmática, controlado posiblemente por la tectónica local.

Desde estos centros eruptivos se emitieron materiales basálticos que dieron lugar a abundantes piroclastos y coladas de lava que llegaron a alcanzar longitu- 


\section{Mapa morfológico de la Montaña del Señalo}
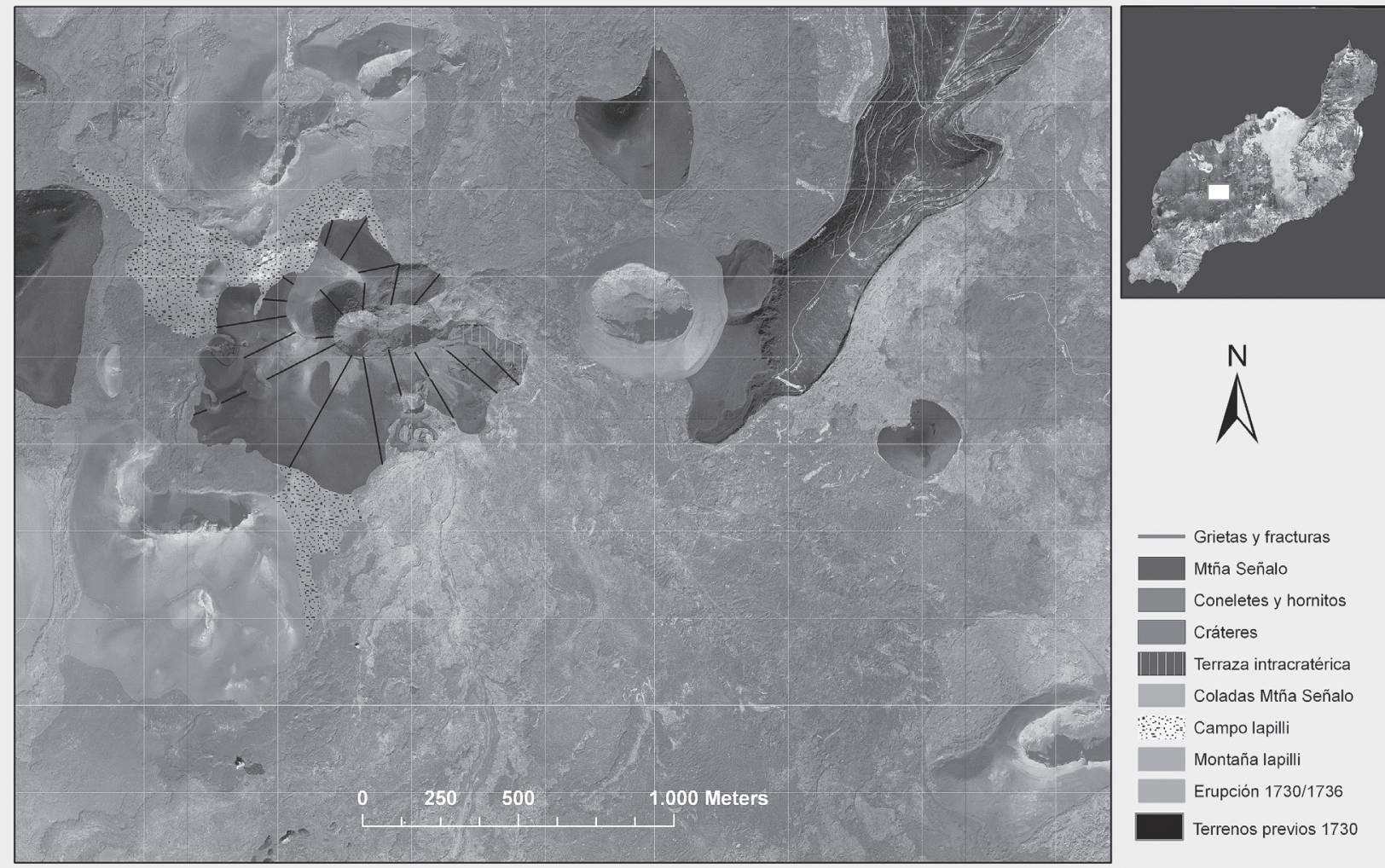

Fig. 2.-Mapa geomorfológico del volcán de Montaña Señalo. Formado por un edificio en forma de herradura abierto hacia el NNE y un conjunto de coneletes y hornitos, a partir de los cuales se desarrollaron las coladas lávicas tanto hacia el norte como hacia el sur.

des máximas de unos $20 \mathrm{~km}$, cubriendo una superficie de alrededor de $200 \mathrm{~km}^{2}$ (Romero et al., 2006).

\section{Montaña Señalo}

El volcán de M. Señalo forma parte del conjunto eruptivo de Pico Partido-M. Señalo. Se trata de una aglomeración constituida por dos edificios yuxtapuestos y parcialmente imbricados de notable envergadura, generados a partir de fracturas paralelas de rumbo ENE-OSO y NO-SE (Romero, 1991).

Montaña del Señalo corresponde a un volcán monogénico (fig. 2) de naturaleza basáltica que desarrolló comportamientos estromboefusivos y está construido a partir de materiales de proyección aérea (lapilli, escorias, spatter, bombas y cenizas) que aparecen soldados hacia la parte superior del cono coincidiendo con los cráteres sumitales. Se trata de un gran edificio en herradura abierto al NO, al que se le superpone un cono volcánico con dos cráteres en embudo y uno abierto al NNE, dotando al conjunto de la complejidad dinámica y morfológica que lo caracteriza (Romero, 1991). Sin embargo, lo más interesante desde el punto de vista volcanomorfológico es la existencia del sistema de cráteres sumitales y la orla basal de hornitos y coneletes escoriáceos (> 10) localizados al SE y al SO del M. Señalo. El cráter en herradura abierto al NNE y todo el sistema de bocas satélites constituyen puntos de salida de lavas muy fluidas, pahoehoe y aa, que alcanzan largos recorridos (Romero et al., 2006) de hasta $14 \mathrm{~km}$ de longitud en el entorno de las Salinas del Janubio (Romero 1991). Todo el conjunto volcánico está afectado por un sistema grietas y fracturas muy desarrolladas vinculadas con procesos de distensión y asentamiento de materiales, en torno a las cuales se constata la existencia de anomalías térmicas superficiales, no activas en la actualidad. 

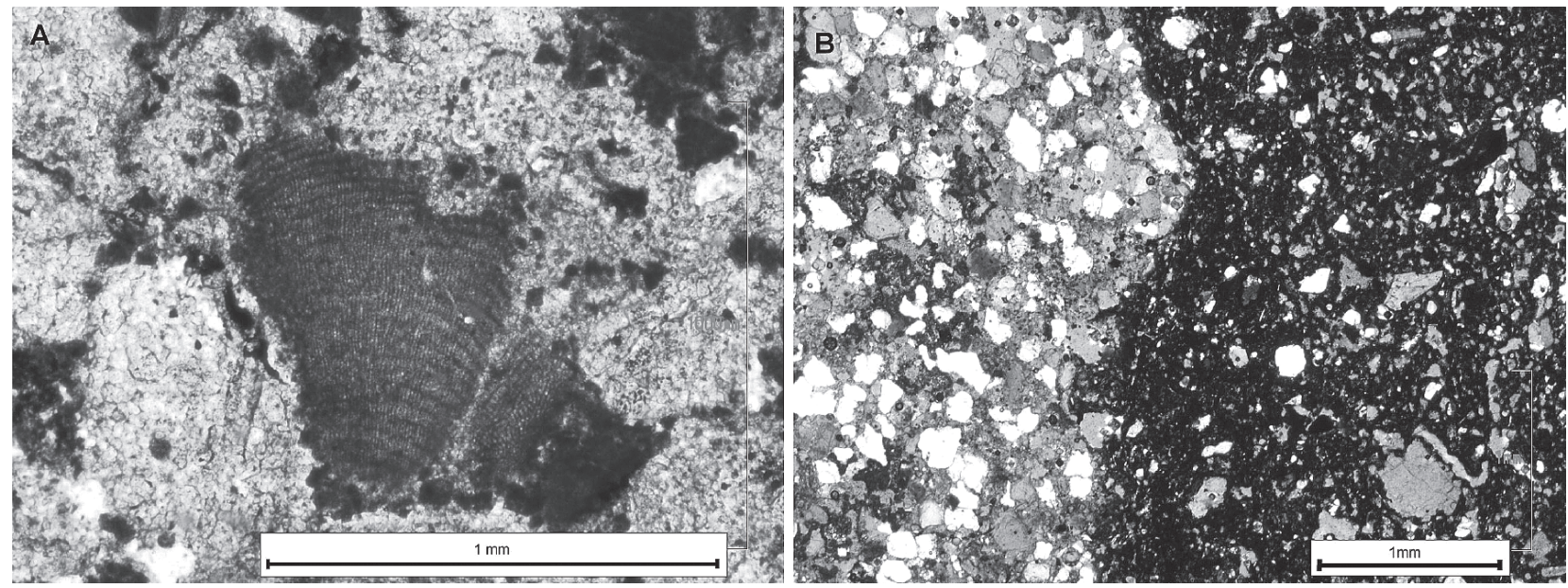

Fig. 3.-Aspecto en lámina delgada que muestra los enclaves sedimentarios de Timanfaya: A, microfotografía de una caliza bioclástica en nícoles paralelos donde se observa la presencia de restos de fosiles; B, microfotografía en nícoles cruzados donde se observa el contacto entre un enclave de arenisca (izda.) y el basalto (dcha.), nótese el descenso en la cristalinidad del basalto en las proximidades del contacto.

Estos materiales emitidos en Montaña Señalo, corresponden con la segunda fase de Carracedo et al. (1990), cuyos magmas toleíticos se caracterizan por la ausencia de enclaves ultramáficos, aumento en la viscosidad además de mecanismos eruptivos más explosivos y menor recorrido de las coladas.

\section{Los enclaves y alteraciones de Montaña Señalo}

Entre las lavas de Timanfaya se reconocen gran cantidad de enclaves de distinta naturaleza, desde origen puramente ígneo hasta propiamente sedimentarios o incluso de origen orgánico además de rocas metamórficas como cuarcitas, pizarras y rocas de silicatos cálcicos. Los enclaves de naturaleza ígnea, principalmente de origen mantélico, han sido ampliamente abordados por Neumann et al. (1995, 2000); Schmincke et al. (1998), entre otros. Sin embargo, los enclaves de naturaleza sedimentaria únicamente han sido considerados en detalle por Araña \& Bustillo (1992), Bustillo et al. (1994) y Aparicio et al. (2006). Estos últimos, describen el origen y composición de metasedimentos englobados en las lavas de Timanfaya, distinguiéndose dos tipos de enclaves: unos de composición silícea cuya mineralogía está compuesta por cuarzo, tridimita y cristobalita, y otros de naturaleza calcosilícea, presentando wollastonita, diópsido y forsterita, además de otros términos intermedios. Esta variación mineralógica se relaciona con procesos metamórficos.
Tras una campaña de reconocimiento de campo en las lavas de la erupción de Timanfaya, se recolectaron varios enclaves entre las coladas de Montaña Señalo que aparecían tanto sueltos como contenidos dentro de las lavas. El análisis petrográfico reveló la presencia de dos tipos litológicos de naturaleza no ígnea: por un lado los más abundantes, presentaban características puramente sedimentarias, dado sus componentes y composición mineralógica, pudiéndose clasificar como lutitas, areniscas y calizas (fig. 3), mientras que los otros, externamente, presentaban un aspecto escoriáceo que rápidamente pasaba hacia el interior a una textura muy vesiculada, de alta porosidad y de colores blanquecinos, sugiriendo por tanto, un origen diferente.

El análisis de lámina delgada de este segundo tipo reveló que en realidad correspondían a dos litologías distintitas (fig. 4), por un lado, el basalto típico de las erupciones de Timanfaya, y por otro una composición aparentemente cuarcífera cuyo contacto con el basalto era gradual en ocasiones y neto en otras. La descripción petrográfica se presenta a continuación.

Los basaltos mostraban una textura porfídica hipocristalina, cuya mineralogía consistía en olivino y plagioclasa principalmente, ya que los clinopiroxenos se disponían en proporciones muy subordinadas. El olivino se presentaba como cristales subidiomorfos inalterados, tanto como fenoristales de dimensiones milimétricas, como mircrolitos en la mesostasia; la plagioclasa por el contrario, presentaba morfologías idiomorfas y se distribuían exclusivamente en la mesostasia sin presentar orientación 


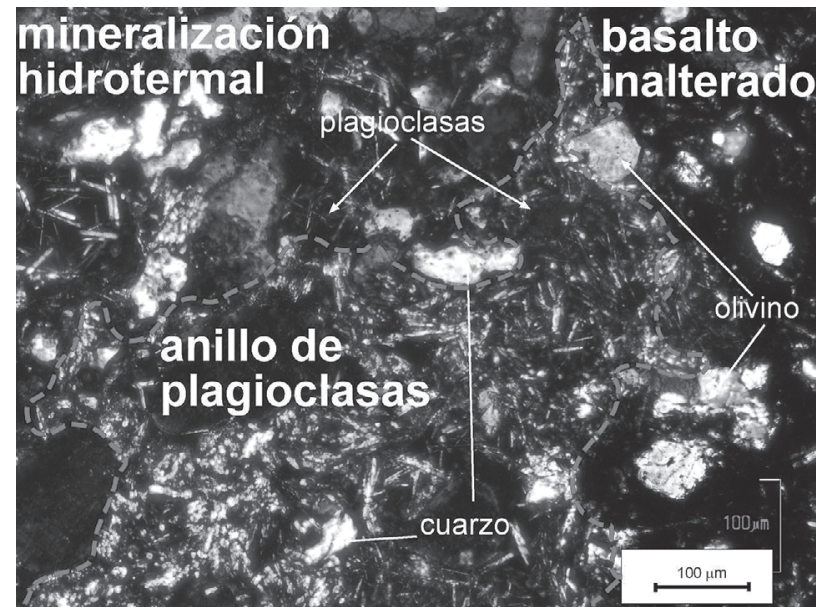

Fig. 4.-Microfotografía en nícoles cruzados de uno de los enclaves hidrotermales. En la parte izquierda se aprecian los granos de cuarzo y los restos de plagioclasas. Nótese cómo aumenta la proporción de esta última conforme nos acercamos al basalto inalterado (dcha.).

ni alteración alguna. Sin embargo, la segunda litología estaba compuesta prácticamente por cristales alotriomorfos de cuarzo de dimensiones muy variables dentro de la misma roca, desde varios milímetros a tamaños submilimétricos. En algunos puntos entre los distintos granos se reconocían algunas plagioclasas.

El contacto entre ambas rocas, como ya se ha comentado anteriormente, en ocasiones era más gradual que en otras, pero para ambos casos presentaba un aumento en la proporción de plagioclasa alrededor, siendo en ocasiones el mineral que marcaba la transición, originándose una especie de anillo submilimétrico a modo de interfase entre ambas litologías (fig. 4). Estas características hacían sospechar que en realidad no se trataba de verdaderos enclaves, sino más bien de algún proceso de alteración.

\section{Análisis por Difracción de Rayos X de las muestras silíceas}

Se realizó un estudio más detallado de la mineralogía de las muestras silíceas mediante Difracción de Rayos X, obteniéndose los resultados recogidos en la tabla 1. Para llevar a cabo este análisis se cortaron y pulverizaron varias muestras para, posteriormente, ser analizadas por el difractor del Museo Nacional de Ciencias Naturales. Si se observa el difractograma de la figura 5 , destacan los principales picos centrados en $3,35,4,26,1,81$ y $59,94 \AA$ $(26,58,20,82,50,10$ y $59,942 \theta)$ del cuarzo, y un

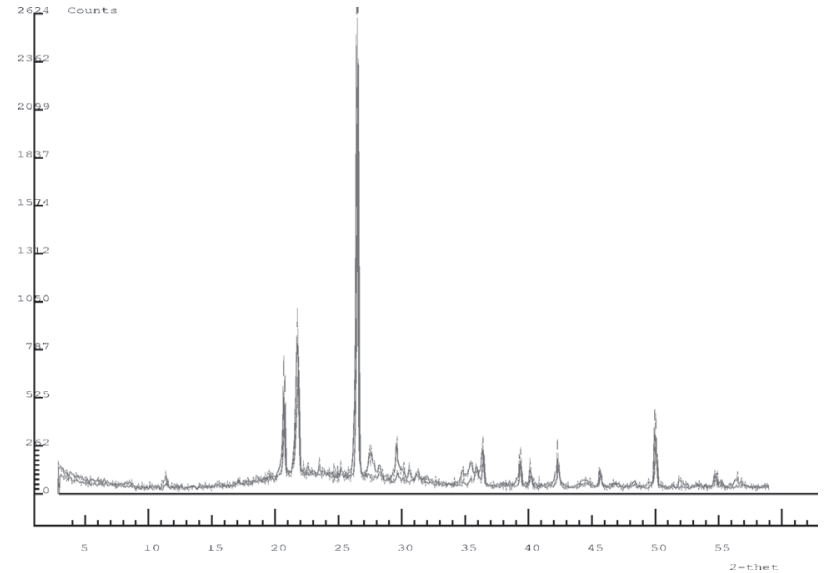

Fig. 5.-Difractogramas obtenidos de los enclaves de naturaleza cuarcífera en contacto con el basalto, explicación en el texto.

Tabla 1.-Porcentaje en peso de las fases minerales detectadas por difracción de rayos $\mathrm{X}$ en los enclaves de naturaleza cuarcífera de Montaña Señalo

\begin{tabular}{lc}
\hline Fase mineral & Porcentaje en peso \\
\hline Cuarzo & $32,00-50,00$ \\
Ópalo (indiferenciado) & $35,50-39,80$ \\
Albita & $1,90-25,70$ \\
Yeso & $6,70-8,30$ \\
\hline
\end{tabular}

pico centrado en 4,05 (21,92 20) que podría corresponder con cristobalita. La presencia de un fondo elevado entre 20-35 20, sugiere la presencia de una fase amorfa tipo opalino. Teniendo en cuenta las distintas fases del ópalo, que varían en función del ordenamiento interno, desde ópalo-A, ópalo-CT a ópalo $\mathrm{C}$, y que esta última presenta unos patrones de difracción muy semejantes a la $\alpha$-cristobalita (polimorfo de baja temperatura), requería un estudio más en detalle para poder hacer una distinción entre ambas.

El problema que se plantea a la hora de discriminar entre la $\alpha$-cristobalita y el ópalo- $C$, es que dado que presenta similares características estructurales, de ahí el nombre de ópalo- $C$, ofrecen espectrogramas muy semejantes y, en ocasiones, son confundidos unos con otros. La presencia de un pico asimétrico en la posición 4,05 junto con otros picos en $3,16,2,84$ y 2,49 hacía sospechar la presencia de esta fase opalina junto con la $\alpha$-cristobalita. Desde que la posición de los picos de 4 Å no puede ser utilizada como herramienta diagnóstica, a la hora de discriminar la $\alpha$-cristobalita de las fases opalinas, Elzea et al. (1994) sugieren el uso del parámetro full width at half maximum (FWHM) o anchura a 
Tabla 2.- Valores de FHWM propuestos en la literatura para cada fase del ópalo y la cristobalita

\begin{tabular}{lccccccc}
\hline \multicolumn{1}{c}{ FWHM } & Ópalo-A & \multicolumn{2}{c}{ Ópalo-CT } & & \multicolumn{2}{c}{ Ópalo-C } & \multicolumn{2}{c}{ Ópalo-CT+C } & Cristobalita \\
\hline Referencia & $(1)$ & $(1)$ & $(2)$ & $(1)$ & $(2)$ & $(2)$ & $(1)$ \\
Mínimo & 3,492 & 0,504 & 0,54 & 0,222 & 0,43 & 0,57 & 0,109 \\
Máximo & 3,492 & 0,883 & 0,69 & 0,453 & 0,57 & 0,61 & 0,140 \\
Media & 3,492 & 0,671 & 0,615 & 0,306 & 0,50 & 0,59 & 0,125 \\
\hline
\end{tabular}

(1) Elzea et al. (1994).

(2) Campbell et al., 2001.

mitad de altura del pico máximo, debido a que las fases opalinas, al presentar un tamaño cristalino menor, mostrarán una línea más ancha que la cristobalita (tabla 2). Otro de los métodos discriminantes propuestos es el calentamiento a $1.050{ }^{\circ} \mathrm{C}$ de la muestra durante $24 \mathrm{~h}$, si se le realiza de nuevo una difracción y aparecen los picos más intensos o con forma ligeramente diferente, indicarían por tanto, que la fase es ópalo-C en lugar de cristobalita, que se mantendría inalterada al calentar, debido a que no experimenta procesos de deshidratación y subsecuente recristalización y aumento de tamaño.

Tampoco la posición del pico de $4 \AA$ es discriminatoria de que haya ópalo-CT y ópalo-C, sino que en todo caso indica la especie más abundante en la muestra (Campbell et al., 2001), de forma que cuando se obtienen valores de FMHM más anchos indica la presencia de ambas fases en la muestra.

Así, si se considera el FWHM como la medida del grado de ordenación de la estructura opalina (Herdianita et al., 2000a, b) resulta difícil la cuantificación de las fases opalinas CT y C si ambas están presentes, recurriéndose al estudio textural de las mismas. El análisis de difracción indicó que el FWHM de las muestras estaba comprendido entre 0,22 y 0,27 , por lo que parece clara la presencia de ópalo-C y por tanto innecesario el segundo método discriminatorio. Así los porcentajes finales para el ópalo-C se estiman del orden del $20 \%$ frente a un fondo de ópalo menos cristalino en proporciones comprendidas entre 10 y $20 \%$.

\section{Microscopio electrónico}

Una vez discriminada la fase opalina con el difractor, se procedió a hacer un estudio con microscopio electrónico de barrido, ya que pese al resultado que indicaba la presencia de opalo- $\mathrm{C}$, el abombamiento entre los 20 y $35^{\circ} 2 \theta$, podría mostrar la presencia de otra fase opalina menos ordenada tipo ópalo-A. Un estudio textural de las distintas morfologías del ópalo se hacía imprescindible a la hora de caracterizar el resto de fases así como su evolución diagenética. Las imágenes obtenidas por el microscopio electrónico de barrido Quanta 200 del Museo Nacional de Ciencias Naturales (fig. 6) mostraban cómo las texturas dominantes correspondían con nanosferas de diámetros inferiores a $1 \mu \mathrm{m}$ que se disponían de forma aislada (fig. 6a), formando agregados de tamaños variables (fig. 6b-c) o en masas más compactas que al ser seccionadas presentaban una estructura interna masiva pero donde todavía se podía reconocer su origen por agregado de nanosferas (fig. 6d). Estas morfologías son las típicas del ópalo A y de la transformación temprana a ópalo CT, por lo que parece clara la presencia de esta fase también en la muestra.

\section{Procedencia de las muestras}

Según el sondeo Lanzarote-1 realizado en la isla durante 1986 de $2.700 \mathrm{~m}$ de profundidad, la base de la isla se localiza a $2.598 \mathrm{~m}$ sobre un paquete sedimentario (Sánchez Guzmán \& Abad, 1986) que según los testigos del sondeo correspondían con areniscas, micritas, arcillas y margas, presentando algunas de ellas microfauna datada en Paleoceno medio y superior. Este paquete sedimentario podría alcanzar espesores del orden de $2 \mathrm{~km}$ según la baja velocidad detectada en perfiles sísmicos realizados en los primeros kilómetros de corteza (Banda et al., 1992).

Así, el origen del primer grupo de enclaves no puede ser otro que el paquete sedimentario bajo la isla dada la presencia de restos de espongiarios y/o restos de algas en las calizas bioclásticas, además de recristalizaciones diagenéticas en el cemento esparítico, aunque no se puede descartar que algunos provengan de paquetes sedimentarios intermedios localizados entre las unidades lávicas de la isla. El contacto de estos enclaves con el basalto estaba marcado por un descenso en el grado de cristalinidad, indicando por tanto que fue el magma el meca- 


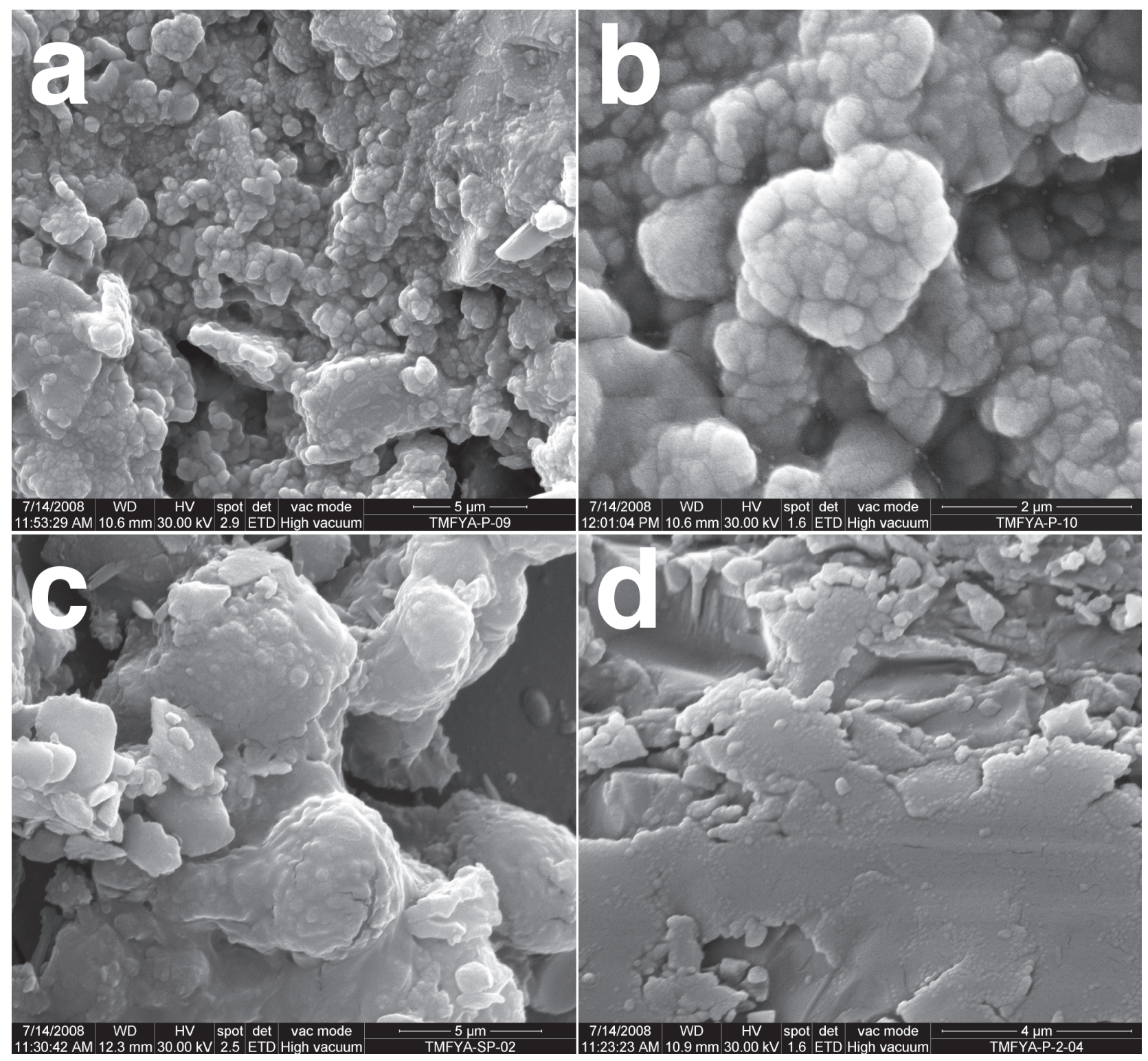

Fig. 6.-Aspecto del ópalo-A al microscopio electrónico de barrido: (a) nanosferas aisladas de ópalo-A recubriendo la superficie del basalto alterado, que van organizándose adquiriendo mayores dimensiones (b) hasta alcanzar tamaños microsféricos del orden de $5 \mu \mathrm{m}$ (c) llegando a formar agregados masivos sin estructura interna pero que se aprecia todavía cómo están formadas por nanosferas (d).

nismo de transporte hacia la superficie, esta característica era especialmente apreciable entre las areniscas donde además mostraban un contacto neto sin signos de asimilación.

Dada la mineralogía y la textura que presentaban las muestras silíceas indicaban que en realidad representaban un proceso de alteración y/o reemplazamiento donde los componentes originales de la roca eran eliminados y sustituidos por otros más estables con las nuevas condiciones termodinámicas, de forma que no constituirían verdaderos encla- ves. La ausencia de los productos típicos de alteración como zeolitas o carbonatos, junto con la mineralogía y las características texturales que presentan tanto el cuarzo como el ópalo y parte de las plagioclasas, hace pensar que corresponden con minerales de neoformación, siendo los procesos hidrotermales los únicos capaces de movilizar estas cantidades de sílice en estos ambientes, sugiriendo por tanto procesos de circulación efectiva de fluidos calientes. Por último, la presencia de yeso se interpreta como un producto secundario de alteración. 
Así, si se tiene en cuenta que podrían representar el producto de una alteración o mineralización hidrotermal, convendría señalar que según este origen no deben ser tratados como enclaves, dado que este término implica la procedencia a partir de otra roca original transportada por el magma a otro punto, por lo que más correctamente deben ser tratados como mineralizaciones o alteración hidrotermal.

Durante la evolución diagenética de la sílice, el ópalo-A es transformado en otras fases más ordenadas tipo ópalo-CT y ópalo-C, culminando este proceso con la formación de cuarzo microcristalino y moganita (Herdianita et al., 2000a). Teniendo en cuenta la juventud de este depósito hidrotermal dado que afecta a las lavas de 1730-1736 (Carmona et al., 2008), y que no procedan de otros basaltos profundos afectados por los mismos procesos y arrastrados por los nuevos aportes magmáticos, sugeriría un proceso mineralizador de menos de 300 años, demasiado corto para producir las transformaciones del ópalo-A a otras formas de la sílice donde al menos 10.000 años de duración son requeridos para la aparición de ópalo CT y más de 50.000 para el cuarzo microcristalino (Rodgers et al., 2004), aunque si la intensidad de flujo es suficiente estos periodos pueden ser reducidos notablemente. Pese a que estos procesos de transformación pueden acelerarse en función de las condiciones y la efectividad de circulación de los fluidos, las características textuales del ópalo de tipo esferas aisladas y en agregados sugiere que correspondan a la primera fase opalina, tal y como se observa en otros sinter modernos compuestos exclusivamente por ópalo-A: Orakei Korako (Smith et al., 2003) y Whakarewarewa (Jones \& Renaut, 2004), ambas en la zona volcánica de Taupo, Nueva Zelanda. La presencia de ópalo C indica por tanto, que la evolución diagenética ha sido bastante rápida, por lo que la intensidad de flujo ha debido ser elevada para la generación de esta última fase sin poder descartarse la acción de la meteorización como agente acelerador de las transformaciones opalinas (Lynne \& Campbell, 2004).

Actualmente, las anomalías geotérmicas en Timanfaya se localizan en los bordes de los cráteres y a favor de fracturas (fig. 7), muestran temperaturas máximas del orden de $600{ }^{\circ} \mathrm{C}$ a tan sólo $12 \mathrm{~m}$ de profundidad en la zona del islote de Hilario y $250{ }^{\circ} \mathrm{C}$ en nivel de superficie, mientras que en zonas alrededor del volcán Timanfaya éstas varían en torno a $150-200{ }^{\circ} \mathrm{C}$ y $80-120{ }^{\circ} \mathrm{C}$ (Araña et al., 1973). La presencia de estas zonas anómalas, muy localizadas, indica la circulación interna efectiva de fluidos calientes, aunque con volúmenes implicados muy reducidos de pocos litros por metro cuadrado, hecho que hace que no existan géiseres en la isla. Según los modelos de trasferencia de calor desarrollado por Araña et al. (1984) y Díez-Gil et al. (1987), estas anomalías están relacionadas con el resto de los conductos que alimentaron la erupción de Timanfaya de 1730-1736, que deben estar todavía a temperaturas superiores a $800{ }^{\circ} \mathrm{C}$ a profundidades en torno a $4 \mathrm{~km}$ (Ortiz et al., 1986). Los primeros estudios y casi los únicos sobre los fluidos, compuestos por agua y $\mathrm{CO}_{2}$ principalmente, que emanaba en estas zonas de anomalía geotérmica, llevados a cabo por Araña \& Panichi (1974), ya sugerían la posibilidad de un sistema hidrológico profundo, de forma que la circulación de fluidos calientes actuaría como mecanismo de transmisión de calor desde las zonas profundas donde se localizaría la fuente térmica hasta la superficie. Los análisis isotópicos de ${ }^{18} \mathrm{O} /{ }^{16} \mathrm{O}$ del vapor de agua sugerían un intercambio isotópico de aguas marinas con rocas oxigenadas en profundidad, que estos autores consideraron que eran las calizas por debajo de la isla, por lo que se ponía de manifiesto las relativas altas profundidades y el origen no magmático de esos fluidos calientes que se observaban en superficie.

Las mineralizaciones hidrotermales han tenido que ser generadas por un fluido sobresaturado en sílice, de forma que teniendo en cuenta la composición de los magmas de la isla proceden de un sistema externo a la misma. Si se considera que composicionalmente estos fluidos no tienen relación con el sistema magmático, las únicas litologías con las características adecuadas para suministrar la sílice son las areniscas del paquete sedimentario y las radiolaritas del fondo oceánico. La disolución del cuarzo en las zonas próximas al foco térmico, haría que la sílice fuera transportada de forma coloidal en forma de $\mathrm{Si}(\mathrm{OH})_{4}$ hasta la superficie, donde el cambio en las condiciones físico-químicas y sobre todo la caída de la temperatura por debajo de los $100{ }^{\circ} \mathrm{C}$ (Lynne et al., 2005) haría que se produjese la coagulación o floculación de los coloides como diferentes nanosferas vítreas de dimensiones inferiores a $500 \mathrm{~nm}$ de diámetro. Este proceso consistiría en un fijado inicial de nanosferas sobre la superficie, donde posteriormente irían incorporándose más nanosferas entre los huecos disponibles, de esta manera la superficie de recubrimiento iría aumentando en espesor (fig. 6d) hasta que en algún caso, pudiesen individualizarse microsferas de mayor tamaño (fig. 6c). 


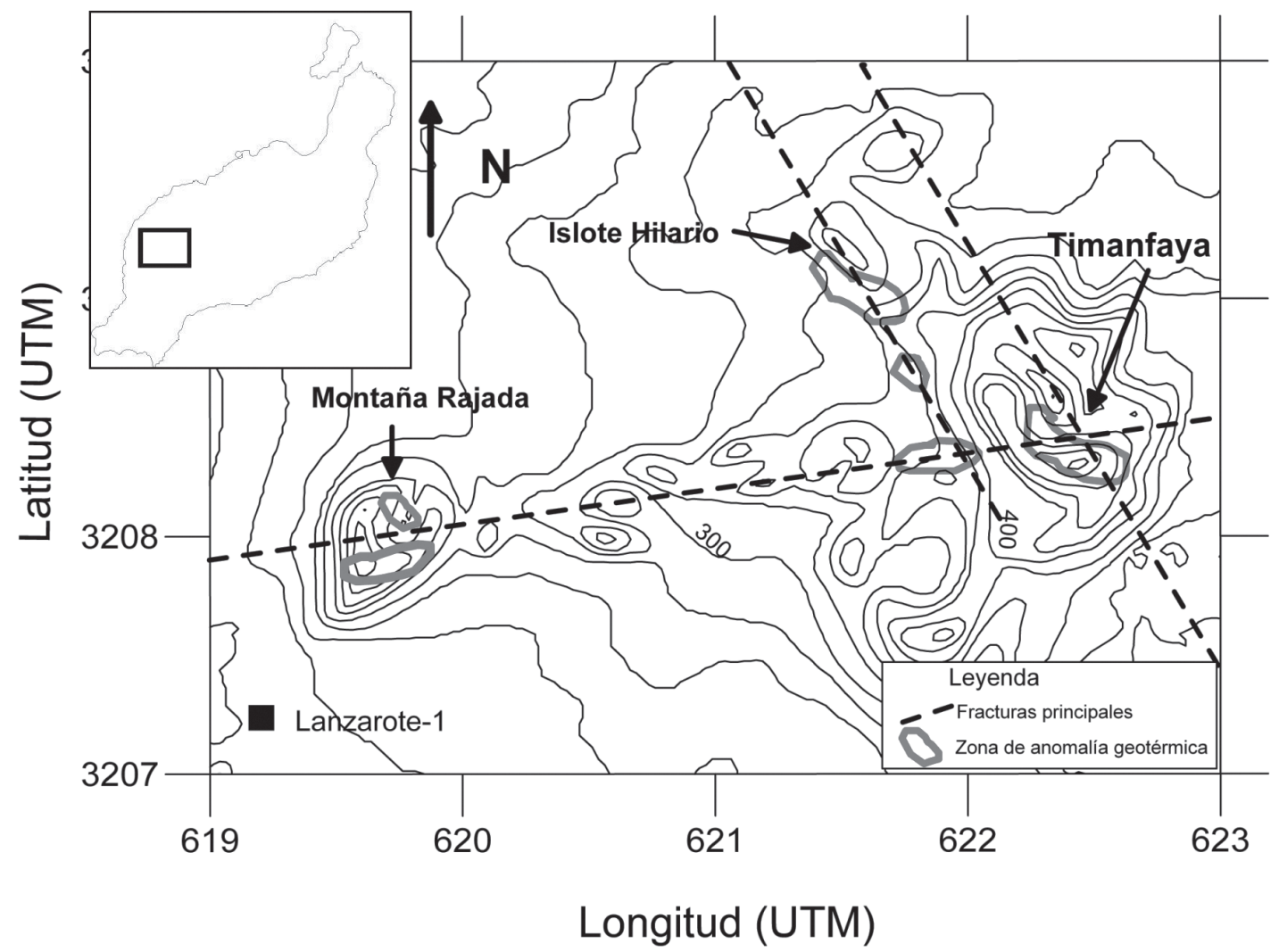

Fig. 7.-Localización de las zonas de anomalía geotérmica en la zona de Montañas de Fuego (Parque Nacional de Timanfaya) a lo largo del sistema de fracturas conjugadas de la erupción de 1730-1736 (modificado de Araña et al., 1984), así como la localización del sondeo geotérmico Lanzarote-1.

\section{Discusión}

Experimentos en laboratorio simulando las condiciones hidrotermales han mostrado que soluciones ácidas silíceas a altas temperaturas y presiones pueden depositar cuarzo a partir de del $\mathrm{Si}(\mathrm{OH})_{4}$, con la cristobalita como fase intermedia (Greenwood, 1967; Carr \& Fyfe, 1958) además de ópalo-A que evolucionará para generar las otras fases diagenéticas. Los fluidos calientes cargados en sílice ascenderían por la columna litológica en forma de coloides, hasta alcanzar la superficie donde se produciría la alteración de los basaltos de la erupción de Timanfaya y se produciría el depósito de ópalo y de cuarzo. Igualmente no se puede descartar que algunas de estas alteraciones encontradas en superficie procedan de basaltos más antiguos afectados por el mismo proceso y que el magma arrastró hacia la superficie.

Por último, cabe mencionar que algunos depósitos hidrotermales de composiciones silíceas, pueden estar asociados a mineralizaciones de sulfuros metá- licos en profundidad; la presencia de mineralizaciones abundantes de pirita entre los 1.818 y los 2.550 m (Sánchez Guzmán \& Abad, 1986) encontradas en el sondeo podría estar asociada a este mismo proceso. Igualmente, futuros estudios sobre los isótopos de oxígeno podrían revelar las condiciones de temperatura en las que estos fluidos silíceos viajaban, además de comprobar la acción de las aguas meteóricas en la formación de las distintas fases opalinas.

\section{Conclusiones}

Entre las lavas de Timanfaya se reconocen dos tipos de enclaves: por un lado, los de naturaleza ígnea, peridotitas en su mayor parte; y por otro, los de naturaleza típicamente sedimentaria: calizas, lutitas y areniscas, además de unos depósitos que sugieren alteraciones de origen hidrotermal que aparecían reemplazando a los basaltos. Este proceso de alteración o mineralización fue llevado a cabo en dos fases, una inicial donde se eliminaron algunos 
componentes originales de la roca, como el olivino y en menor proporción las plagioclasas, y otra donde se produjo el depósito de la fase hidrotermal.

Estos depósitos están compuestos en su mayor parte por ópalo y cuarzo, el estudio textural sugiere que la fase amorfa dominante sea ópalo-A, aunque los valores de FWHM indican claramente la presencia de ópalo-C con porcentajes de hasta el $20 \%$. Como estas alteraciones afectan a las lavas de la erupción de 1730-1736, indica que se trata de un proceso mineralizador de menos de 300 años y, por tanto, la textura esperable del ópalo sea exclusivamente nanosferas y microsferas de ópalo-A como única fase amorfa, puesto que la evolución diagenética de esta fase está fuertemente condicionada por el tiempo transcurrido, las condiciones de circulación de fluidos y la acción meteórica. La presencia de ópalo-C sugiere que esta mineralización ha sido afectada enormemente por estos últimos procesos, acelerándose así la generación de este tipo de fases en la evolución diagenética del ópalo.

Teniendo en cuenta la presencia de un sistema profundo de circulación de aguas, se produciría la movilización de la sílice desde las zonas profundas y calientes, en forma coloidal, hasta la superficie donde durante su ascenso irían interaccionando con las rocas que atravesase. Con este mecanismo se justificaría la presencia de depósitos hidrotermales en superficie con el propio sistema hidrológico de la isla. Igualmente la presencia de alteraciones en zonas relativamente alejadas de las actuales anomalías geotérmicas sugiere la presencia en el pasado de otras que posiblemente se encuentren ahora selladas.

\section{AGRADECIMIENTOS}

Nos gustaría agradecer a Alfredo Aparicio sus constructivas críticas y sus buenos consejos en la elaboración y mejora de este trabajo, igualmente a María Ángeles Bustillo por su ayuda prestada en la interpretación y comprensión de los difractogramas. Igualmente al proyecto de investigación del CSIC 200430E438.

\section{Referencias}

Abdel-Monem, A.; Watkins, N.D. \& Gast, P.W. (1971). Potassium-Argon ages, volcanic stratigraphy, and geomagnetic polarity history of the Canary Islands: Lanzarote, Fuerteventura, Gran Canaria and La Gomera. American Journal of Science, 271: 490-521.

Aparicio, A.; Bustillo, M.A.; García, R. \& Araña, V. (2006). Metasedimentary xenoliths in the lavas of the Timanfaya eruption (1730-1736), Lanzarote, Canary
Islands): metamorphism and contamination processes. Geological Magazine, 143: 181-193.

doi:10.1017/S0016756806001713

Araña, V. \& Bustillo, M.A. (1992). Volcanologic concerns of the silicious metasedimentary xenoliths included in historic lava-flows of Lanzarote (Canary Islands). Acta Volcanológica, 2: 1-6.

Araña, V.; Díez, J.L.; Ortiz, R. \& Yuguero, J. (1984). Convection of Geothermal Fluids in the Timanfaya Volcanic Area (Lanzarote, Canary Islands). Bulletin Volcanologique, 47: 667-677. doi:10.1007/BF01961234

Banda, E.; Danobeitia, J.J.; Surinach, E. \& Ansorge, J. (1981). Features of crustal structure under the Canary Islands. Earth and Planetary Science Letters, 55: 11-24. doi:10.1016/0012-821X(81)90082-0

Araña, V. \& Panichi, C. (1974). Isotopic composition of steam samples from Lanzarote, Canary Islands. Geothermics, 3: 142-145. doi:10.1016/0375-6505(74)90012-1

Araña, V.; Ortiz, R. \& Yuguero, J. (1973). Thermal anomalies in Lanzarote (Canary Islands). Geothermics, 2: 73-75. doi:10.1016/0375-6505(73)90006-0

Bustillo, M.A.; Nishimura, A.; Araña, V. \& Hattori, I. (1994). Paleocene radiolarians from Xenoliths hosted in Holocene lavas of Lanzarote (Canary Islands). Geobios, 27: 181-188. doi:10.1016/S0016-6995(94)80005-7

Campbell, K.A.; Sannazzaro, K.; Rodgers, K.A.; Herdianita, N.R. \& Browne, P.N.L. (2001). Sedimentary facies and mineralogy of the Late Pleistocene Umukuri silica sinter, Taupo volcanic zone, New Zealand. Journal of Sedimentary Research, 71: 727-746.

Carmona, J.; García, A.; Romero, C. \& Dóniz, J. (2008). New evidences of a double hydrological system in Lanzarote (Canary Islands). Resúmenes de la $6^{\underline{a}}$ Asamblea Hispano Portuguesa de Geodesia y Geofísica, Tomar (Portugal).

Carracedo, J.C. \& Badiola, E. (1991). Lanzarote. La erupción volcánica de 1930. CSIC-Estación volcanológica de Canarias, 184 pp.

Carracedo, J.C.; Rodríguez Badiola, E. \& Soler, V. (1990). Aspectos volcanológicos y estructurales. Evolución e implicaciones en riesgo volcánico de la erupción de 1730 en Lanzarote. Islas Canarias. Estudios Geológicos, 46: 25-55. doi:10.3989/egeol.90461-2436

Carr, R.M. \& Fyfe, W.S. (1958) Some observations on the crystallization of amorphous silica. American Mineralogist, 43: 908-916.

Coello, J.; Cantagrel, J.M.; Hernan, F.; Fuster, J.M.; Ibarrola, E.; Ancochea, E.; Casquet, C.; Jamond, C.; Díaz de Terán, J.R. \& Cendrero, A. (1992). Evolution of the eastern volcanic ridge of the Canary Islands based on new K-Ar data. Journal of Volcanology and Geothermal Research, 53: 251-274. doi:10.1016/0377-0273(92)90085-R

Díez Gil J.L.; Araña, V.; Ortiz R. \& Yuguero, J. (1987). Stationary convection model for heat transport by means of geothermal fluids in post eruptive systems. Geothermics, 15: 77-87. doi:10.1016/0375-6505(87)90080-0

Elzea, J.M.; Odom, I.E. \& Miles, W.J. (1994). Distinguishing well ordered opal-CT and opal-C from high 
temperature cristobalite by x-ray diffraction. Analytica Chimica Acta, 286: 107-116. doi:10.1016/0003-2670(94)80182-7

Flörke, O.W.; Graetsch, H.; Martin, K.; Roller, K. \& Wirth, R. (1991). Nomenclature of micro- and noncrystalline silica minerals, based on structure and microstructure. Neues Jahrbuch für Mineralogie Abhandlungen, 163: 19-42.

Fournier, R.O. \& Rowe, J.J. (1966). Estimation of underground temperatures from the silica content of water form hot springs and wet-steam wells. American Journal of Science, 264: 685-697.

Fúster, J.M.; Fernández Santín, S. \& Sagredo, J. (1968). Geología y volcanología de las Islas Canarias. Lanzarote. Instituto Lucas Mallada, CSIC, Madrid, 239 pp.

Greenwood, R. (1967) Thermal behavior of SiO2-X and its relation to the natural silica minerals. American Mineralogist, 52: 1662-1668.

Herdianita, N.R.; Browne, P.R.L. Rodgers, K.A. \& Campbell, K.A. (2000a). Mineralogical and textural changes accompanying ageing of silica sinters. Mineralium Deposita, 35: 48-62. doi:10.1007/s001260050005

Herdianita, N.R.; Rodgers, K.A. \& Browne, P.R.L. (2000b). Routine instrumental procedures to characterise the mineralogy of modern and ancient silica sinters. Geothermics, 29: 65-81. doi:10.1016/S03756505(99)00054-1

Ibarrola, E.; Cantagrel, J.M.; Fúster, J.M.; Coello, J. \& Jamond, C. (1988). Geocronología de las series volcánicas neógenas de Lanzarote (Islas Canarias). Actas simposio sobre volcanismo, SGE, 345-348.

Jones, B. \& Renaut, R.W. (2004). Water content of opal-A: implications for the origin of laminae in geyserite and sinter. Journal of Sedimentary Research, 67: 88-104.

Jones, B. \& Segnit, E.R. (1971). The Nature of opal: nomenclature and constituent phases. Journal of the Geological Society of Autralia, 18: 57-68.

Neumann, E.-R.; Sorensen, V.B.; Simonsen, S.L. \& Johnsen, K. (2000). Gabbroic xenoliths from La Palma, Tenerife and Lanzarote, Canary Islands: evidence for reactions between mafic alkaline Canary Islands melts and old oceanic crust. Journal of Volcanology and Geothermal Research, 103: 313-342. doi:10.1016/S0377-0273(00)00229-8

Neumann, E.-R.; Wulff-Pedersen, E.; Johnsen, K.; Andersen, T. \& Krogh, E. (1995). Petrogenesis of spinel harzburgite and dunite suite xenoliths from Lanzarote, eas- tern Canary Islands: Implications for the upper mantle. Lithos, 35: 83-107. doi:10.1016/0024-4937(95)91153-Z

Lynne, B.; Campbell, Y.; Kathleen A.; Moore, J.N. \& Browne, P.R.L. (2005). Diagenesis of 1900-year-old siliceous sinter (opal-A to quartz) at Opal Mound, Roosevelt Hot Springs, Utah, U.S.A. Sedimentary Geology, 179: 249-278. doi:10.1016/j.sedgeo.2005.05.012

Ortiz, R.; Araña, V.; Astiz, M. \& García, A. (1986). Magnetotelluric study of the Teide (Tenerife) and Timanfaya (Lanzarote) volcanic areas. Journal of Volcanology and Geothermal Research, 30: 357-377. doi:10.1016/0377-0273(86)90061-2

Rodgers, K.A.; Browne, P.R.L.; Buddle, T.F.; Cook, K.L.; Greatrex, R.A.; Hampton, W.A.; Herdianita, N.R.; Holland, G.R.; Lynne, B.Y.; Martin, R.; Newton, Z.; Pastars, D.; Sannazarro, K.L. \& Teece, C.I.A. (2004). Silica phases in sinters and residues from geothermal fields of New Zealand. Earth-Science Reviews, 66: 1-61. doi:10.1016/j.earscirev.2003.10.001

Romero, C. (1991). La erupción de Timanfaya (Lanzarote, 1730-1736). Análisis documental y estudio geomorfológico. Servicio de Publicaciones ULL, Sta. Cruz de Tenerife, $136 \mathrm{pp}$.

Romero, C.; Dóniz Páez, J.; García, L. \& Amado, P. (2006). Significado Volcanológico de los Depósitos de Spatter de la Erupción Volcánica de 1730-1736 de Timanfaya (Lanzarote. Canarias. España). Proceedings 5a Asamblea Hispano-Portuguesa de Geodesia y Geofísica. Sevilla. España. (CD, ISBN: 84-8320-373-1).

Sánchez-Guzmán, J. \& Abad, J. (1986). Sondeo geotérmico Lanzarote-1. Anales de Física, serie B, 82: 102-109.

Schmincke, H.U.; Klugel, A.; Hansteen, T.H.; Hoernle, K. \& van den Bogaard, P. (1998). Samples from the Jurassic ocean crust beneath Gran Canaria, La Palma and Lanzarote (Canary Islands). Earth and Planetary Science Letters, 163: 343-360. doi:10.1016/S0012-821X(98)00168-X

Smith, B.Y.; Turner, S.J. \& Rodgers, K.A. (2003). OpalA and associated microbes form Wairakei, New Zealand: the first 300 days. Mineralogical Magazine, 67: 563-579. doi:10.1180/0026461036730118

Smith, D.K. (1998). Opal, cristobalite and tridymite: noncrystallinity versus crystallinity, nomenclature of the silica minerals and bibliography. Powder Diffraction, 13: 2-19.

Recibido el 18 de septiembre de 2008 Aceptado el 13 de febrero de 2009 Publicado online el 23 de abril de 2009 